\title{
Fabrication of Fresnel zone plates by holography in the extreme ultraviolet region
}

\author{
Sankha S. Sarkar ${ }^{\mathrm{a})}$ and Pratap K. Sahoo \\ Laboratory for Micro- and Nanotechnology, Paul Scherrer Institute, 5232 Villigen PSI, Switzerland \\ Harun H. Solak ${ }^{\text {b) }}$ \\ Laboratory for Micro- and Nanotechnology, Paul Scherrer Institute, 5232 Villigen PSI, Switzerland and \\ EULITHA AG, 5232 Villigen PSI, Switzerland \\ Christian David and J. Friso Van der Veen \\ Laboratory for Micro- and Nanotechnology, Paul Scherrer Institute, 5232 Villigen PSI, Switzerland
}

(Received 18 June 2008; accepted 25 August 2008; published 1 December 2008)

\begin{abstract}
Fabrication of Fresnel zone plates (FZPs) with nanometer scale resolution is one of the major challenges in lithographic fabrication. The authors present a holographic approach to create FZPs where the interference pattern between a spherical beam and a plane wave is recorded to obtain the FZP structure. The spherical beam is obtained by diffraction from a circular aperture (pinhole) in a semiopaque membrane which is illuminated by a spatially coherent extreme ultraviolet beam. The beam transmitted by the membrane serves as the reference plane wave. The resulting pattern gives rise to a FZP with outermost zone width comparable to the dimension of the circular aperture. Fabrication and test results of a FZP obtained with this method are presented. The technique offers a solution to the pattern-placement problem encountered in serial ZP writing techniques as well as high resolution potential. (C) 2008 American Vacuum Society. [DOI: 10.1116/1.2987960]
\end{abstract}

\section{INTRODUCTION}

Focusing of $\mathrm{x}$ rays is of great interest for studies of the chemical, magnetic, structural, electronic, and elemental properties of materials at the nanometer scale. In addition, the ability of $x$ rays to penetrate deep into matter allows one to probe buried materials. Weak refraction and high absorption complicate the use of conventional optics (lenses and mirrors) in this spectral region.

Considerable interest has grown in the use of diffractive optical elements, Fresnel zone plates (FZPs) being the most common of them. FZPs consist of $N$ concentric circles with varying width where the radius $r_{n}$ of the $n$th ring is given by ${ }^{1}$

$$
r_{n}^{2}=n \lambda f+\frac{n^{2} \lambda^{2}}{4},
$$

where $\lambda$ is the wavelength of radiation concerned and $f$ is the corresponding first order focal length. Commonly the resolution of the zone plate is given by the spot size of the first order focus and it is of the order of the smallest feature size of the zone plate which is the outermost zone width $\left(\Delta r_{n}\right)$.

Currently high resolution FZPs are almost exclusively fabricated by electron beam lithography (EBL) ${ }^{2,3}$ This fabrication method is challenged by difficulties in the placement of the pattern, the proximity effect, and problems related to finite pixel size and reproducibility. First holographic technique of ZP fabrication at extreme ultraviolet (EUV) regime was demonstrated by Solak et al. ${ }^{4}$ In this article, we introduce a holographic technique that overcomes these difficul-

\footnotetext{
${ }^{a)}$ Electronic mail: sankha.sarkar@psi.ch

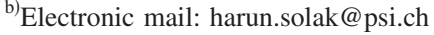

ties. EUV lithography offers sub-50 nm scale pattern fabrication possibility and hence high resolution potential.

The principle of the holographic approach, as illustrated in Fig. 1, is to let the diverging spherical wave obtained by diffraction from a circular aperture (pinhole) interfere with a plane wave of comparable intensity. The spherical wave is obtained by the diffraction of a plane wave through a pinhole. The pinhole is fabricated on a semitransparent membrane which also allows the plane wave to pass through, with an attenuated magnitude. The interference between these two waves gives rise to a hologram of the pinhole which is essentially a zone plate pattern. The pattern is then recorded at a certain distance $z$ from the pinhole in a sensitive resist, and subsequently the pattern is transferred to a suitable thin film in order to obtain a working zone plate.

A simple analysis shows that the interference between a spherical phase front $\left(e^{i \vec{k} \cdot \vec{r}}\right)(\vec{k}$ being the wave vector) and a plane wave front $\left(e^{i \vec{k} \cdot \vec{z}}\right)$ gives rise to corresponding minima or maxima that follows the relation presented in Eq. (1). The diffracted spherical wave field amplitude decreases as $1 / r, r$ being the distance of the observation point in the exposure plane from the pinhole. The relative intensity between the diffracted wave and the plane reference wave is controlled by the diameter of the pinhole, the thickness of the membrane on which the pinhole is fabricated, and the separation between the pinhole and the exposure plane $(z)$. At EUV and $\mathrm{x}$-ray wavelength most materials have complex refractive index,

$$
n=1-\delta+i \beta,
$$

where $\delta$ is responsible for phase shift and $\beta$ represents absorption of the field amplitude in the material. Since the 


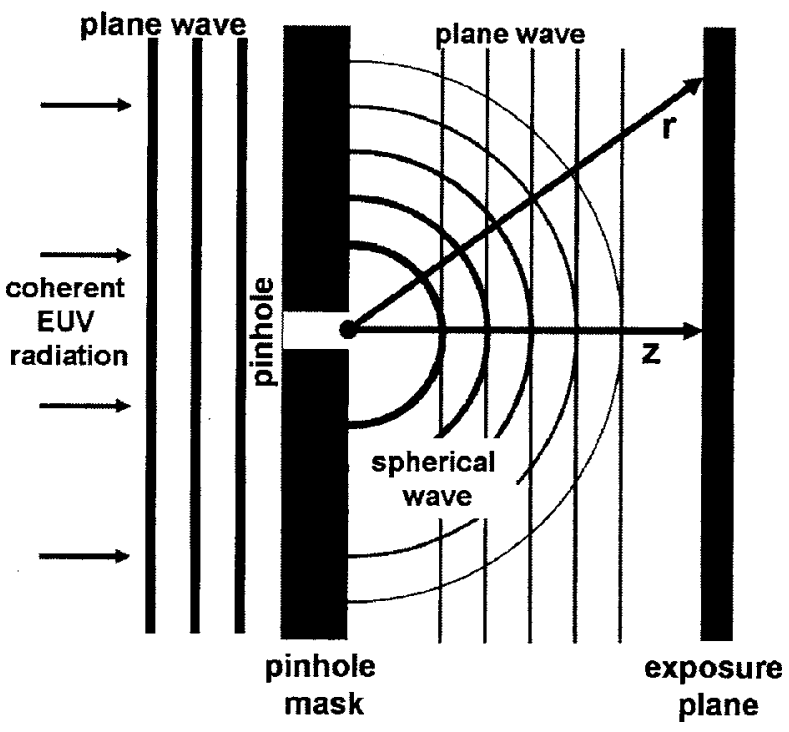

FIG. 1. Schematic of the holographic technique. Spherical wave created by pinhole diffraction interferes with the attenuated plane wave partially transmitted through the pinhole mask to produce FZP pattern on the exposure plane at a distance $z$

spherical beam created by a small pinhole has very low intensity, a highly absorbing metal is deposited on the membrane in order to achieve comparable relative intensities.

\section{EXPERIMENTAL DETAILS}

The pinhole is fabricated by electron beam lithography (EBL) on a silicon nitride $\left(\mathrm{Si}_{3} \mathrm{~N}_{4}\right)$ membrane (Fig. 2). A $100 \mathrm{~nm}$ thick membrane with a $23 \mathrm{~nm}$ thick chromium hard mask layer on the top is coated with $80 \mathrm{~nm}$ thick poly(methyl methacrylate) (PMMA) resist and exposed [Fig. 2(a)] with electron beam using LION LV1 lithography machine. The exposed resist is removed with a 1:3 solution of isopropyl alcohol: methyl isobutyl ketone. Subsequently the circular hole pattern [Fig. 2(b)] is transferred into the $\mathrm{Cr}$ hard mask by reactive ion etching (RIE) using chlorine plasma [Fig. 2(b)]. After removing the remaining resist using oxygen $\left(\mathrm{O}_{2}\right)$ plasma, the pinhole is then etched through the silicon nitride membrane by RIE using plasma of a mixture of hexaflurosulphide $\left(\mathrm{SF}_{6}\right)$ and trifluromethane $\left(\mathrm{CHF}_{3}\right)$ [Fig. 2(c)]. Additional $160 \mathrm{~nm}$ of gold $(\mathrm{Au})$ is deposited on the top [Fig. 2(d)]. The diameter of the pinhole after Au deposition is measured to be $300 \mathrm{~nm}$.

The membrane containing the pinhole is used as a mask for EUV holographic exposure performed at X-ray Interference Lithography ${ }^{5}$ (XIL) beamline at the Swiss Light Source (SLS). The mask is placed in the path of a spatially coherent EUV plane wave of $13.4 \mathrm{~nm}$ wavelength (transverse coherence length $^{6}$ of $\sim 2.1 \mathrm{~mm}$ ) with $2.5 \%$ bandwidth. The pinhole diffracts the EUV radiation which then interferes with the attenuated plane reference wave leaking through the rest of the membrane. The resulting pattern is recorded on another $\mathrm{Cr}(23 \mathrm{~nm})$ coated silicon nitride membrane $(300 \mathrm{~nm}$ thick) [Fig. 3(a)] placed at a distance of $300 \mu \mathrm{m}$ from the parent mask in a sensitive chemically amplified EUV resist
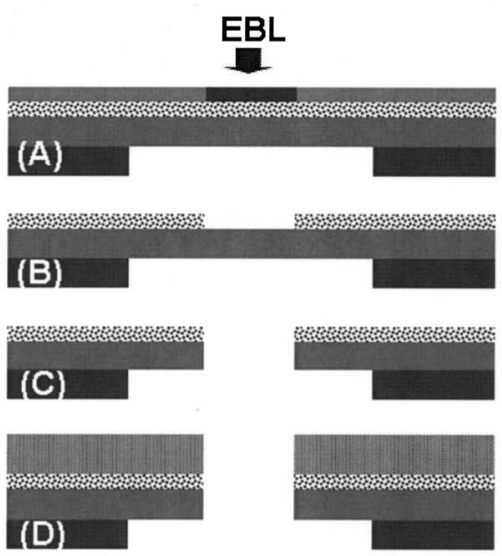

$\mathrm{Si} \square \mathrm{Si}_{3} \mathrm{~N}_{4}$ 䌦 $\mathrm{Cr}$ resist $\mathrm{Au}$

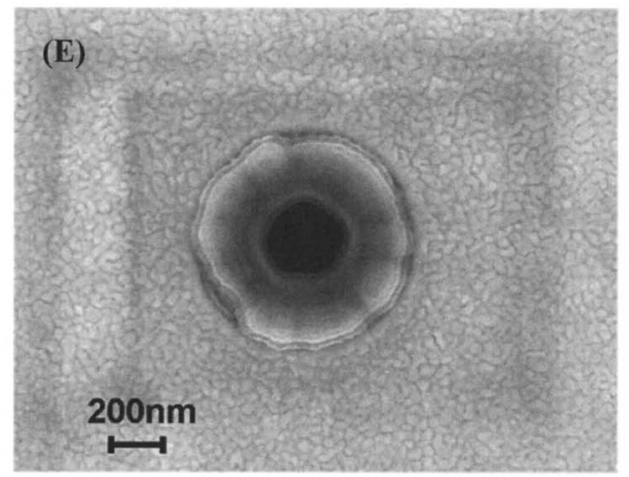

FIG. 2. (A)-(D) Schematic of the pinhole mask fabrication. Starting with a $\mathrm{Cr}(23 \mathrm{~nm})$ coated $\mathrm{Si}_{3} \mathrm{~N}_{4}(100 \mathrm{~nm})$ membrane on $\mathrm{Si}$ substrate, EBL exposure is done on PMMA layer on the membrane (A). After development the $\mathrm{Cr}$ is etched with $\mathrm{Cl}_{2}$ plasma (B), and subsequently $\mathrm{Si}_{3} \mathrm{~N}_{4}$ is etched through (C) and additional $\mathrm{Au}(160 \mathrm{~nm})$ is deposited (D). (E) shows scanning electron micrograph of a pinhole with $300 \mathrm{~nm}$ diameter.

MET 2D (Ref. 7) (Rohm and Haas). The plane wave is attenuated to $0.012 \%$ transmission due to the absorption by the membrane and the $\mathrm{Cr}$ and $\mathrm{Au}$ layers. Hence the total transmitted intensity is extremely low and the choice of highly sensitive resist is crucial. MET 2D provides higher sensitivity with dose to clear below $20 \mathrm{~mJ} / \mathrm{cm}^{2}$. With the beamline flux set to be at $40 \mathrm{~mW} / \mathrm{cm}^{2}$, the typical exposure time is of the order of $30 \mathrm{~min}$. After exposure the resist is baked for $90 \mathrm{~s}$ at $120{ }^{\circ} \mathrm{C}$ and subsequently developed with developer MF26A (Rohm and Haas) for $60 \mathrm{~s}$.

$\mathrm{ZP}$ pattern is visible with optical microscope after resist development. The pattern is transferred into the $\mathrm{Cr}$ film by RIE [Fig. 3(b)]. Subsequently $\mathrm{Cr}$ is used as a hard mask to etch into the $\mathrm{Si}_{3} \mathrm{~N}_{4}$ membrane by about $60 \mathrm{~nm}$ [Fig. 3(c)] using the RIE process mentioned earlier. The later etch step is included in order to achieve higher diffraction efficiency by the fabricated FZP. Finally using a photolithographic exposure step the rest of the membrane is covered with a thick resist (Shipley 1813) layer leaving the FZP of interest open and covering everything else on the rest of the membrane [Fig. 3(d)]. A scanning electron microscopy (SEM) image of the structure is shown in Fig. 3(e) (taken before photolithog- 
(A)

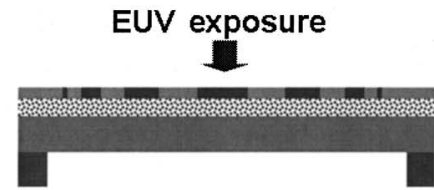

(B)

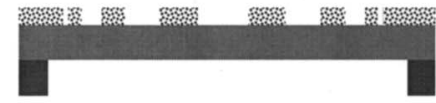

(C)

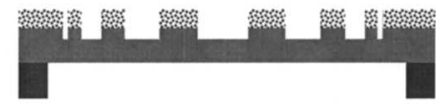

(D)

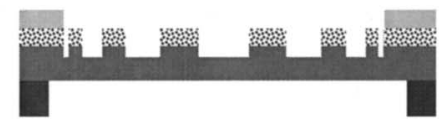

si
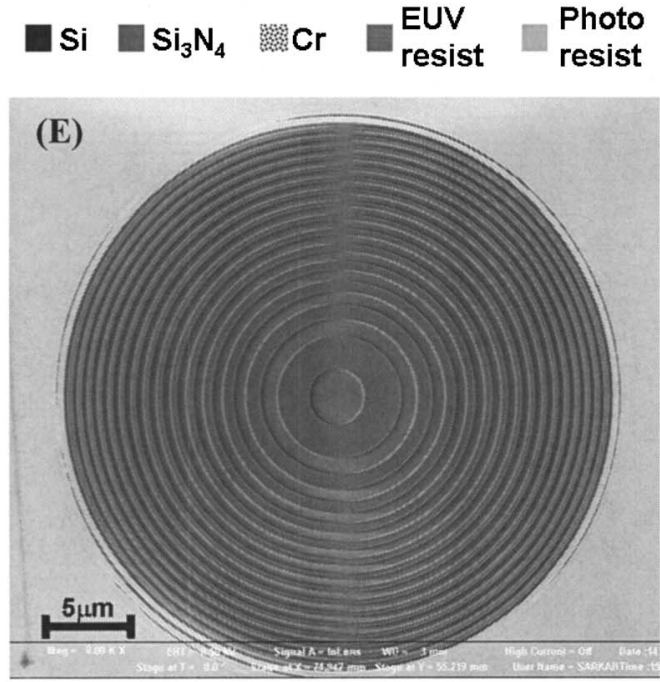

FIG. 3. (A)-(D) Schematic of the FZP processing technique. Starting with a $\mathrm{Cr}(23 \mathrm{~nm})$ coated $\mathrm{Si}_{3} \mathrm{~N}_{4}(100 \mathrm{~nm})$ membrane on $\mathrm{Si}$ substrate, the holographic intensity pattern is recorded on the EUV resist coated membrane (A) and after development $\mathrm{Cr}$ is etched to transfer the $\mathrm{ZP}$ pattern (B). $\mathrm{Si}_{3} \mathrm{~N}_{4}$ is etched partially $(\mathrm{C})$ and finally a photolithography is done in order to cover the rest of the membrane (D). (E) shows a scanning electron microscopic image of the FZP produced with the pinhole shown in Fig. 2(e).

raphy step). The diameter of the FZP is measured to be $32 \mu \mathrm{m}$ and the number of zones is 30 , the outermost smallest feature being $260 \mathrm{~nm}$. The micrograph shows an apparent discontinuity (or phase shift) in the zones along a vertical line that goes across the zones, especially in the top half of the picture. A careful look reveals the fact that the zones are continuous and the apparent phase shift is probably an artifact due to a dynamical charging effect on the insulating $\mathrm{Si}_{3} \mathrm{~N}_{4}$ membrane, as the electron beam is scanned across the structure. We are convinced of the above argument as this feature on the top part of the image remains at the same place even when the sample is rotated under the microscope.

The radius of the concentric circular rings [Fig. 3(e)] is measured from the SEM image and square of that is plotted as a function of the zone index in Fig. 4, which shows the validity of the ZP pattern obtained by the holographic process. The deviation of the measured zone radius from the ideal zone radius (obtained from the slope of the linear fit to

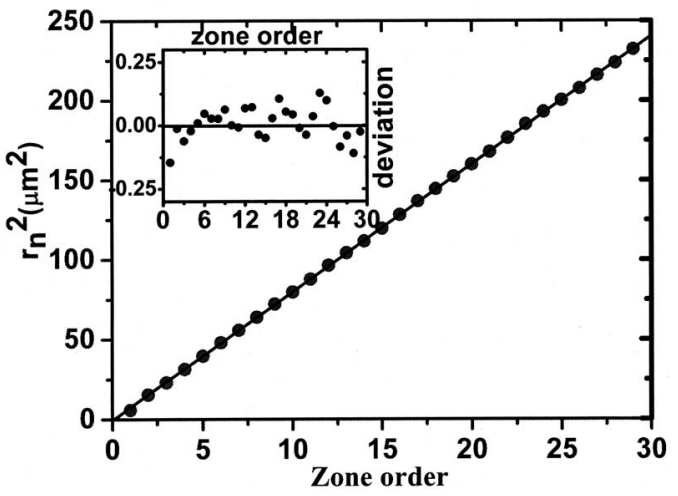

FIG. 4. Zone radius measured from the SEM image (squared) is plotted with zone number (green circles). The straight line fit shows a focal length of $610 \mu \mathrm{m}$. The inset shows the zone placement deviation normalized to the local zone width.

Fig. 4) normalized to the local zone width gives a measure of the local zone deviation (Fig. 4 inset). The finite deviation is attributed to inaccuracies in the zone radius measurement technique which is limited by the finite pixel size of the SEM micrograph.

The focal spot size of the zone plate is measured at the same wavelength at the same beamline. The membrane containing the zone plate is placed in the path of the EUV plane wave. A knife edge scan is performed through the focal spot. A photodiode is placed behind the knife edge to measure the intensity as the scan is performed. The knife edge is fabricated on another silicon nitride membrane coated with a $80 \mathrm{~nm} \mathrm{Cr}$ layer. The thick $\mathrm{Cr}$ edge provides high contrast for the measurement. The measured knife edge scan profile is presented in Fig. 5. Theoretically the integrated intensity profile at the focal plane of a lens of same numerical aperture as of the zone plate is calculated and compared with the measurement (Fig. 5). The focal spot size is measured to be $300 \mathrm{~nm}$ (distance between $90 \%$ and 10\% of the intensity profile $^{8}$ ) which is comparable to both the outermost zone width $(260 \mathrm{~nm})$ and the diameter of the pinhole $(300 \mathrm{~nm})$. The seemingly perfect agreement between the theoretically

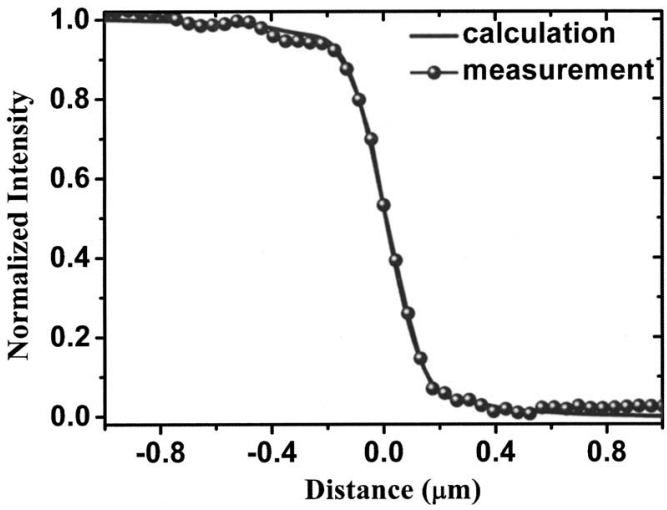

FIG. 5. Knife edge scan profile of the zone plate focal spot. The (black) dotted line represents the measured profile, while the (blue) solid line represents the calculated theoretical behavior. 
calculated and experimentally measured curves is a testament to the aberration-free nature of the holographically made FZP.

\section{DISCUSSION}

The properties of the obtained FZP are determined by the properties of the incident radiation and the pinhole mask. First, the longitudinal coherence length of the incident beam which is related to its spectral bandwidth $(\Delta \lambda)$ limits the optical path length difference that is allowed between the spherical and plane waves. This condition effectively places a limit on the number of zones that can be printed using radiation of a certain spectral width. The spectral width can be reduced and hence the number of zones increased by the use of a monochromator, although one has to consider the impact on the exposure time.

The minimum zone width, on the other hand, is related to the size of the diffracting aperture. For a circular pinhole, the usable part of diffraction is limited to the zeroth order lobe in the Airy pattern. ${ }^{9}$ Therefore in order to diffract to higher angles and to make higher resolution FZP one needs to use a smaller aperture. However, from a practical point of view one also has to consider the impact of a smaller diffracting aperture on the exposure time. The intensity of light diffracted by a circular aperture at a distance $z$ is proportional to $\left(d^{2} / \lambda z\right)^{2}$, where $d$ is the diameter of the aperture. This formula represents the tradeoff between different parameters. For example, if we are interested in keeping the exposure time constant and reducing the zonewidth by a factor of 2 , we can achieve this by reducing the pinhole diameter by a factor of 2 and the distance $z$ by a factor of 4 . While the FZP obtained in this way will have the desired zonewidth, its radius will be half of the original. A straightforward derivation based on above arguments reveals the following relation which puts a limit on the number $(N)$ of zones that can be fabricated by this technique as a function of the incident photon flux $(F)$, photoresist sensitivity $(S)$, and maximum feasible exposure time $\left(t_{\mathrm{ex}}\right)$,
$N=\sqrt{\frac{4 \pi^{2} F t_{\mathrm{ex}}}{0.14 S}}$.

It is interesting to note that the practical limit on exposure time does not directly limit the resolution of the FZP that can be obtained by this method. Instead one has to compromise between resolution and the FZP diameter. However, in order to increase the number of zones one has to utilize either a more powerful beam or a more sensitive photoresist.

In conclusion, we have demonstrated a holographic technique where a FZP is fabricated by recording the interference pattern formed by a spherical and plane wave. The experimental performance agrees well with theoretical calculations. Fabrication using EUV light offers sub-50 nm resolution potential due to the short wavelength and the absence of photoelectron caused proximity effect in this energy range.

\section{ACKNOWLEDGMENTS}

The authors would like to thank the Swiss National Science Foundation for financial support. The holographic exposures and measurements have been performed at the Swiss Light Source, Paul Scherrer Institut, Villigen, Switzerland.

${ }^{1}$ M. Young, J. Opt. Soc. Am. 62, 972 (1972).

${ }^{2}$ W. Chao, B. D. Harteneck, J. A. Liddle, E. H. Anderson, and D. T. Attwood, Nature (London) 435, 1210 (2005).

${ }^{3}$ K. Jefimovs, J. Vila-Comamala, T. Pilvi, J. Raabe, M. Ritala, and C. David, Phys. Rev. Lett. 99, 264801 (2007).

${ }^{4}$ H. H. Solak, C. David, and J. Gobrecht, Appl. Phys. Lett. 85, 2700 (2004).

${ }^{5}$ H. H. Solak, J. Phys. D 39, R171 (2006).

${ }^{6}$ Coherence length calculation uses the treatment given in F. van der Veen and F. Pfeiffer, J. Phys.: Condens. Matter 16, 5003 (2004).

${ }^{7}$ J. W. Thackeray et al., J. Photopolym. Sci. Technol. 20, 411 (2007).

${ }^{8}$ D. Atwood, Soft X-rays and Extreme Ultraviolet radiation: Principles and Applications (Cambridge University Press, Cambridge, 2000), p. 359.

${ }^{9} \mathrm{~J}$. W. Goodman, Introduction to Fourier Optics (Roberts, Colorado, 2005). 